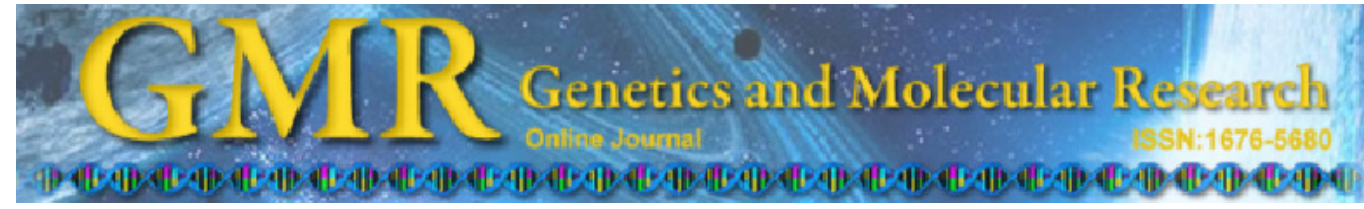

\title{
Two splice variants of the bovine lactoferrin gene identified in Staphylococcus aureus isolated from mastitis in dairy cattle
}

\author{
J.M. Huang, Z.Y. Wang, Z.H. Ju, C.F. Wang, Q.L. Li, T. Sun, Q.L. Hou, \\ S.Q. Hang, M.H. Hou and J.F. Zhong \\ Center of Dairy Cattle Research, Shandong Academy of Agricultural Sciences, \\ Jinan, P.R. China \\ Corresponding author: J.F. Zhong \\ E-mail: huangjinm@sina.com \\ Genet. Mol. Res. 10 (4): 3199-3203 (2011) \\ Received January 17, 2011 \\ Accepted June 20, 2011 \\ Published December 21, 2011 \\ DOI http://dx.doi.org/10.4238/2011.December.21.1
}

\begin{abstract}
Bovine lactoferrin (bLF) is a member of the transferrin family; it plays an important role in the innate immune response. We identified novel splice variants of the $b L F$ gene in mastitis-infected and healthy cows. Reverse transcription-polymerase chain reaction (RT-PCR) and clone sequencing analysis were used to screen the splice variants of the $b L F$ gene in the mammary gland, spleen and liver tissues. One main transcript corresponding to the $b L F$ reference sequence was found in three tissues in both healthy and mastitis-infected cows. Quantitative real-time PCR analysis showed that the expression levels of the $L F$ gene's main transcript were not significantly different in tissues from healthy versus mastitis-infected cows. However, the new splice variant, LF-AS2, which has the exon-skipping alternative splicing pattern, was only identified in mammary glands infected with Staphylococcus aureus. Sequencing analysis showed that the new splice variant was 251 bp in length, including exon 1, part of exon 2, part of exon 16 , and exon 17 . We conclude that $b L F$ may play a role in resistance to mastitis through alternative splicing mechanisms.
\end{abstract}

Key words: Dairy cattle; Lactoferrin gene; Alternative splicing; Mastitis 


\section{INTRODUCTION}

Bovine mastitis is an inflammation of the mammary gland caused by microorganisms and is a topic of major preoccupation for the dairy industry worldwide. Over 100 different microorganisms, usually bacteria, can cause mastitis and these vary greatly in the route by which they reach the cow and in the nature of the disease they cause. Staphylococcus aureus is generally recognized as a common udder pathogen of bovine mastitis, causing herd problems and significant economic losses for the producer and the dairy industry. Several epidemiological studies have shown that the prevalence of the clinical mastitis caused by $S$. aureus ranged from 12.3 to $55.2 \%$ in different countries (Nickerson et al., 1995; Pitkala et al., 2004; Nagahata et al., 2007).

Lactoferrin (LF), also known as LTF, is a member of the transferrin family and is found in milk, bile, saliva, tears, and polymorphonuclear cell granules (Baker and Baker, 2005). Much attention, especially regarding polymorphisms and expression (Pawlik et al., 2009; Huang et al., 2010), has been focused on the $L F$ gene due to its many functions, including antibacterial, antiviral, antifungal, anti-inflammatory, anti-oxidant, and immunomodulatory activities (Ward et al., 2005). The bovine LF (bLF) concentration increases during mammary involution and infection (Hurley and Rejman, 1993; Komine et al., 2005). Accordingly, $b L F$ has been considered an important component in mammary gland innate defense mechanisms against mastitis. It has been reported that clinical mastitis causes a significant increase in $L F$ gene expression in dairy cattle (Kawai et al., 1999; Chaneton et al., 2008; Swanson et al., 2009). Distinct invasion and pathogenic mechanisms of different types of mastitis caused by different organisms have been reported and therefore are likely to produce dissimilar immune reactions. In fact, different immune responses have been associated with udder infected with S. aureus, S. uberis and Escherichia coli (Hagiwara et al., 2003; Bannerman et al., 2004; Wellnitz and Kerr, 2004; Swanson et al., 2009). However, the underlying mechanism of the $b L F$ gene regulation response to distinct pathogens is not yet well understood.

Alternative splicing (AS) is the molecular process that allows a single gene to produce many gene products or proteins with potentially different functions. AS is a cellular mechanism for determining diversity in animals and is a well-established regulatory mechanism for gene expression. Unlike promoter activity, which primarily regulates the amount of transcripts, AS changes the structure (Stamm et al., 2005). Bovine immune response associated with mastitis is a very complex biological process. We hypothesized that the $b L F$ gene plays its role through the AS mechanism, allowing it to adapt the complexity of the mammary gland immune response to different pathogens. Therefore, the present study is focused on the identification of splice variants of the $L F$ gene in healthy and mastitis-infected cows.

\section{MATERIAL AND METHODS}

\section{Identification of $b L F$ gene splice variants}

Total RNA was isolated from the mammary gland tissue, liver and spleen of the mastitis and healthy cows by TRIZOL kit (Sango Biothec, Shanghai, China). cDNA was obtained from RNA using the PrimerScript RT Master Mix kit (TaKaRa, Dalian, China). Then, $1 \mu \mathrm{L}$ cDNA and the Lf primers (F: 5'-TGGATAAAGGGACGCAGAAC-3'; R: 5'-GGAGGCAG 
GCTAGCTTCTTT-3'; product size $=2223 \mathrm{bp}$; annealing termperature $=60^{\circ} \mathrm{C}$ ) were used as template to amplify the coding region of the $b L F$ gene with the TaKaRa LA Taq kit. The PCR product was purified and linked to the PMD18-T vector. Thirty positive clones were sequenced. The sequence alignments were carried out by the DNAMAN software. A Bos taurus lactotransferrin gene mRNA reference sequence (NM_180998.2) and genomic sequence (NC_007320.3) deposited in NCBI were used as a reference. The splice variant was confirmed by resequencing the clone twice.

\section{Gene expression of the main transcript of the $b L F$ gene}

Quantitative real-time PCR (Q-PCR) was used to compare $b L F$ mRNA expressions of 5 healthy and 5 mastitis tissue samples. Q-PCR was carried out using cDNA templates and the SYBRGreen PCR Master Mix (Tiangen, China) in BioRad CFX96 Real-Time System according to the manufacture protocol. Each sample was run in triplicate. Q-PCR primers of the $b L F$ gene (F: 5'-TCCAGACTCTGTGCCTTGTG-3'; R: 5'-TGTTCTCCCAGACTGTGTCG-3'; product size $=160 \mathrm{bp}$; annealing temperature $\left.=60^{\circ} \mathrm{C}\right)$ and $\beta$-actin gene $\left(\mathrm{F}: 5^{\prime}\right.$-GCACAATGAAGATCAA GATCATC-3'; R: 5'-CTAACAGTCCGCCTAGAAGCA-3'; product size = $173 \mathrm{bp}$; annealing temperature $=60^{\circ} \mathrm{C}$ ). The relative quantification of $b L F$ gene expression was calculated by the standard curve-based method for relative real-time PCR as described by Larionov et al. (2005).

\section{RESULTS AND DISCUSSION}

Q-PCR results showed no significant difference between the $b L F$ mRNA expression found for healthy and mastitis cow tissues $(\mathrm{P}>0.05)$. Bovine Affymetrix Bovine Genome Array analysis in our laboratory also obtained a similar result (data not shown). Our finding is not consistent with previous reports (Kawai et al., 1999; Chaneton et al., 2008; Swanson et al., 2009).

A functional immune system would rely heavily on several mechanisms of gene regulation to achieve the required diversity and flexibility of function. Recently, much attention has focused on understanding the crucial role of transcriptional regulation in the immune system; however, much less attention has been given to another widespread mechanism of gene regulation, namely AS (Lynch, 2004). Interestingly, 11 splice variants encoding 7 different proteins have been found in the human $L F$ gene (www.ensembl.org). To gain a better understanding of the $b L F$ gene regulation, AS was investigated in three tissues in healthy and mastitis cows. The $b L F$ gene is located on bovine chromosome 22, contains 17 exons and spans about 34.5 $\mathrm{kbp}$ of genomic DNA. The complete coding region of the $b L F$ gene includes $2127 \mathrm{bp}$ and encodes 708 -amino acid proteins. The coding region was amplified by designing the primers in the exon 1 untranslated region and 3'untranslated region. A 2223-bp fragment of the $b L f$ gene and the control gene fragment were amplified in the mammary gland, spleen and liver tissues in healthy and mastitis cows by RT-PCR (Figure 1). The size and sequence of amplicons were identical to each corresponding target region of the $b L F$ gene. The other faint fragment (251 bp) was only found in the mammary gland of the mastitis cow. To further identify its sequence, PCR product was used for the clone sequencing, which yielded 162 clones of LF-AS1 and the other two clones were LF-AS2. Based on the frequencies of isoforms, the LF-complete is considered to be the major transcript; whereas, the frequency of LF-AS2 was $1.23 \%(2 / 162)$, 
a low frequency transcript. Sequence alignment results showed that LF-AS1 is identical to the B. taurus lactotransferrin gene mRNA reference sequence (NM_180998.2) in NCBI. LFAS2 consists of exon 1, partial exon 2, partial exon 16, and exon $\overline{17}$, while other sequences were spliced out (Figure 2). The splicing pattern of LF-AS2 is an exon skipping event. The identity of the LF-AS2 is $10.17 \%$ as compared with the LF-complete. Two important domains (transferrin) in 25-352 and 364-693 amino acids are found in the $b L F$ gene (http://pfam. sanger.ac.uk/). The deletion in LF-AS2 can result in the lacking of domain of the $b L F$ protein. Furthermore, recent publications have shown that numerous immunologically relevant genes have been found to undergo alternative splicing, having related, distinct or even opposing functions in spite of their abundance (Garcia-Blanco et al., 2004; Lynch, 2004). In the present study, the novel spliced variant LF-AS2 of the $b L F$ gene may exercise different functions in the mammary gland. Further studies remain to be done to confirm the presence of LF-AS2 at the protein level and to clarify its function during the immune response in mastitis cow.

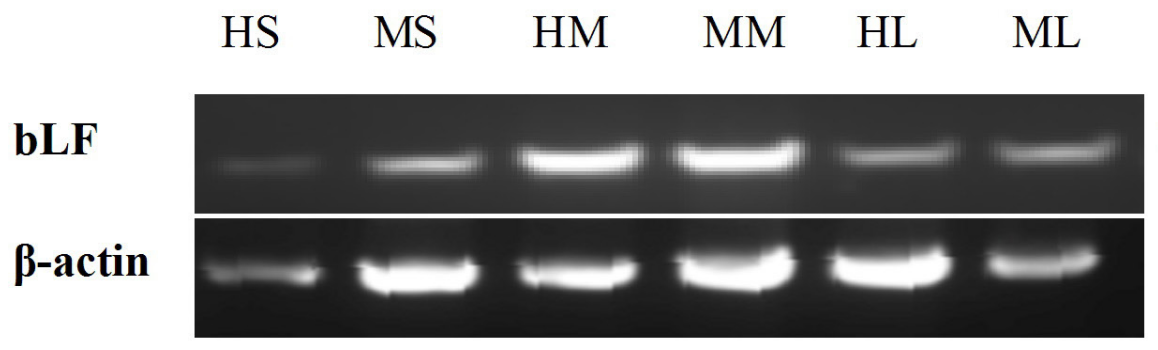

$2223 \mathrm{bp}$

$173 \mathrm{bp}$

Figure 1. RT-PCR results of $b L F$ and $\beta$-actin genes in healthy and mastitis cows. HS = healthy cow spleen; MS = mastitis cow spleen; $\mathrm{HM}$ = healthy cow mammary gland; $\mathrm{MM}=$ mastitis cow mammary gland; $\mathrm{HL}$ = healthy cow liver; $\mathrm{ML}=$ mastitis cow liver.

\section{A LF-AS1 (LF-complete)}

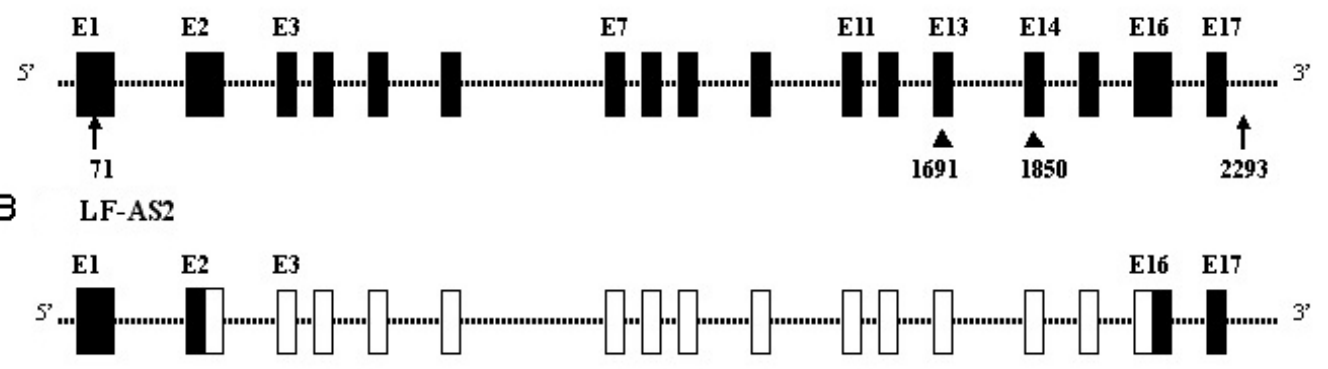

Figure 2. Sketch map of $L F$ gene splice variants. A. A total of $2223 \mathrm{bp}$, which consists of 17 exons. B. A 251-bp fragment including exon 1, partial exon 2, partial exon 16, and exon 17. E-exon: black boxes represent exon; white boxes represent deletion sequence; black triangles indicate the position of bLF Q-PCR primers; arrows show the positions of primers for the amplification of the $b L F$ gene coding region. 


\section{ACKNOWLEDGMENTS}

Research supported by grants from the National Natural Science Foundation of China (\#31000543), the Support Program of the Ministry of Science and Technology, P.R. China (\#2011BAD19B02), the Major Project of National Transgene in China (\#2011ZX08007-001), the Program of National Cow Industrial Technology System (\#CARS-37), the Youth Science Foundation from Shandong Academy of Agriculture Science (\#2006YQN030), the Projects of the Department of Science and Technology of Shandong Province (\#2007LZ10-04), and the Natural Science Foundation of Shandong Province (\#Y2007D72).

\section{REFERENCES}

Baker EN and Baker HM (2005). Molecular structure, binding properties and dynamics of lactoferrin. Cell Mol. Life Sci. 62: 2531-2539.

Bannerman DD, Paape MJ, Lee JW, Zhao X, et al. (2004). Escherichia coli and Staphylococcus aureus elicit differential innate immune responses following intramammary infection. Clin. Diagn. Lab. Immunol. 11: 463-472.

Chaneton L, Tirante L, Maito J, Chaves J, et al. (2008). Relationship between milk lactoferrin and etiological agent in the mastitic bovine mammary gland. J. Dairy Sci. 91: 1865-1873.

Garcia-Blanco MA, Baraniak AP and Lasda EL (2004). Alternative splicing in disease and therapy. Nat. Biotechnol. 22: 535-546.

Hagiwara S, Kawai K, Anri A and Nagahata H (2003). Lactoferrin concentrations in milk from normal and subclinical mastitic cows. J. Vet. Med. Sci. 65: 319-323.

Huang JM, Wang HM, Wang CF, Li JB, et al. (2010). Single nucleotide polymorphisms, haplotypes and combined genotypes of lactoferrin gene and their associations with mastitis in Chinese Holstein cattle. Mol. Bio. Rep. 37: 477483.

Hurley WL and Rejman JJ (1993). Bovine lactoferrin in involuting mammary tissue. Cell Biol. Int. 17: 283-289.

Kawai K, Hagiwara S, Anri A and Nagahata H (1999). Lactoferrin concentration in milk of bovine clinical mastitis. Vet. Res. Commun. 23: 391-398.

Komine K, Komine Y, Kuroishi T, Kobayashi J, et al. (2005). Small molecule lactoferrin with an inflammatory effect but no apparent antibacterial activity in mastitic mammary gland secretion. J. Vet. Med. Sci 67: 667-677.

Larionov A, Krause A and Miller W (2005). A standard curve based method for relative real time PCR data processing. BMC Bioinformatics 6: 62.

Lynch KW (2004). Consequences of regulated pre-mRNA splicing in the immune system. Nat. Rev. Immunol. 4: 931-940.

Nagahata H, Ito H, Maruta H, Nishikawa Y, et al. (2007). Controlling highly prevalent Staphylococcus aureus mastitis from the dairy farm. J. Vet. Med. Sci. 69: 893-898.

Nickerson SC, Owens WE and Boddie RL (1995). Mastitis in dairy heifers: initial studies on prevalence and control. $J$. Dairy Sci. 78: 1607-1618.

Pawlik A, Sender G and Korwin-Kossakowska A (2009). Bovine lactoferrin gene polymorphism and expression in relation to mastitis resistance-a review. Anim. Sci. Pap. Rep. 27: 263-271.

Pitkala A, Haveri M, Pyorala S, Myllys V, et al. (2004). Bovine mastitis in Finland 2001 - prevalence, distribution of bacteria, and antimicrobial resistance. J. Dairy Sci. 87: 2433-2441.

Stamm S, Ben-Ari S, Rafalska I, Tang Y, et al. (2005). Function of alternative splicing. Gene 344: 1-20.

Swanson KM, Stelwagen K, Dobson J, Henderson HV, et al. (2009). Transcriptome profiling of Streptococcus uberisinduced mastitis reveals fundamental differences between immune gene expression in the mammary gland and in a primary cell culture model. J. Dairy Sci. 92: 117-129.

Ward PP, Paz E and Conneely OM (2005). Multifunctional roles of lactoferrin: a critical overview. Cell Mol. Life Sci. 62: 2540-2548.

Wellnitz O and Kerr DE (2004). Cryopreserved bovine mammary cells to model epithelial response to infection. Vet. Immunol. Immunopathol. 101: 191-202. 